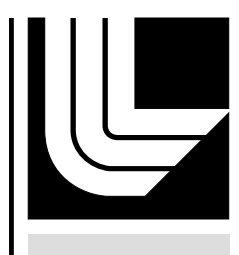

LAWRENCE LIVERMORE N A TION A L LABORATORY

\title{
UCRL-TR-210179
}

\section{Ultrasonic NDE of Multilayered Structures}

M. J. Quarry, K. A. Fisher, S. K. Lehman

March 3, 2005 
This document was prepared as an account of work sponsored by an agency of the United States Government. Neither the United States Government nor the University of California nor any of their employees, makes any warranty, express or implied, or assumes any legal liability or responsibility for the accuracy, completeness, or usefulness of any information, apparatus, product, or process disclosed, or represents that its use would not infringe privately owned rights. Reference herein to any specific commercial product, process, or service by trade name, trademark, manufacturer, or otherwise, does not necessarily constitute or imply its endorsement, recommendation, or favoring by the United States Government or the University of California. The views and opinions of authors expressed herein do not necessarily state or reflect those of the United States Government or the University of California, and shall not be used for advertising or product endorsement purposes.

This work was performed under the auspices of the U.S. Department of Energy by University of California, Lawrence Livermore National Laboratory under Contract W-7405-Eng-48. 


\section{FY04 LDRD Final Report Ultrasonic NDE of Multilayered Structures LDRD Project Tracking Code: 02-ERD-010 Michael Quarry, Principal Investigator}

\section{Overview}

This project developed ultrasonic nondestructive evaluation techniques based on guided and bulk waves in multilayered structures using arrays. First, a guided wave technique was developed by preferentially exciting dominant modes with energy in the layer of interest via an ultrasonic array. Second, a bulk wave technique uses Fermat's principle of least time as well as wave-based properties to reconstruct array data and image the multilayered structure.

The guided wave technique enables the inspection of inaccessible areas of a multilayered structure without disassembling it. Guided waves propagate using the multilayer as a waveguide into the inaccessible areas from an accessible position. Inspecting multi-layered structures with a guided wave relies on exciting modes with sufficient energy in the layer of interest. Multilayered structures are modeled to determine the possible modes and their distribution of energy across the thickness. Suitable modes were determined and excited by designing arrays with the proper element spacing and frequency.

Bulk wave imaging algorithms were developed to overcome the difficulties of multiple reflections and refractions at interfaces. Reconstruction algorithms were developed to detect and localize flaws. A bent-ray algorithm incorporates Fermat's principle to correct time delays in the ultrasonic data that result from the difference in wave speeds in each layer and refractions at the interfaces. A planar wave-based algorithm was developed using the Green function for the multilayer structure to enhance focusing on reception for improved imaging.

\section{Relevance to Lab Mission}

Both guided and bulk wave inspection techniques developed in this work will enhance LLNL's role of Stockpile Stewardship and inspecting weapons without disassembly. Experiments have also shown guided wave's scalability to smaller curvatures, such as boiler tubing, and shows promise for application to NIF targets. In addition, the planar multilayer diffraction tomography reconstruction algorithms may image density distributions in explosives as well as shallow, near earth, environments to look for buried hazardous waste and covert trans-border tunnels.

\section{Introduction/Background}

\section{Guided Waves}

Ultrasonic guided waves are a powerful new technology for nondestructive evaluation. Conventional ultrasound uses bulk waves that are generated by a sensor that 
is often scanned across a part to produce an image. With guided waves structures such as plates, tubes, and rods are utilized as waveguides. A sensor generates a mode from a single position on the waveguide, and the mode propagates along the waveguide. Echoes of the mode arriving back at the sensor give an indication of damage. Guided wave modes are analogous to light waves that propagate in fiber optics. Guided waves can propagate distances of hundreds of feet to inspect large areas quickly and into inaccessible areas such as buried pipes or embedded parts of a weapon. These properties make guided waves an effective technology for many applications.

Guided wave inspection has been well established in a single layer, such as in piping and tubing, to inspect long distances from a single inspection point [1,2]. Many modes may exist in the structure, and phase and group velocity dispersion curves must be analytically calculated for a structure to characterize the dispersive nature of the waveguide. Group velocity is the speed at which the mode wave packet propagates along the waveguide and can be used to localize any damage indications. Each mode is unique in its sensitivity and ability to propagate, since it has a unique displacement pattern and energy distribution across the thickness. Exciting a mode with optimal sensitivity is essential to successful application of guided waves and is achieved through sensor design.

The research of this project was to study the application of guided waves to multiple layers and develop array sensors to excite and detect modes suitable for inspecting layers of interest. To inspect a layer of interest a sensor must excite a mode with most of its energy in the layer of interest. Suitable modes for inspecting a given layer can be identified by modeling the dispersion curves. Arrays can then be designed with proper element spacing and frequency to investigate the prospective modes and experimentally determine the optimum sensitivity.

\section{$\underline{\text { Bulk Waves }}$}

Typically, a tomographic reconstruction is derived from a series of projections, where a projection is defined as the integral of the image in the direction specified by that angle. Traditional methods of acoustic/seismic tomography rely on algorithms that can generate a two-dimensional velocity map from a set of line integrals representing the travel time of numerous rays throughout the medium of interest. Most algorithms use straight-ray back projections to reconstruct the medium velocity, density, or attenuation.

The motivation for our development is driven by the need to detect and image flaws in multilayered structures. In many multilayered structures there are large contrast differences in the acoustic velocities and impedances between adjacent layers resulting in significant refraction effects. Multiple reflections between interfaces and attenuation are also problems that result from changes in the acoustic velocities of the layers. With prior knowledge of the internal structure algorithms can be developed to overcome the difficulties created by the interfaces.

Research in this project focused on developing inverse wave imaging algorithms to identify deviations, defects, and or flaws in the assembled multilayered structure. One algorithm employs Fermat's principle of least time to determine the appropriate ray path from the transmitter to scatterer (flaw) to receiver. This equates to a bent-ray correction

to the collected ultrasonic data in contrast to more typical straight ray assumptions. The 
approximation of our forward model admits a single scattering event from a point scatterer, which provides a method for estimating changes in refractive index. Another algorithm was developed using a planar multilayer Green function to utilize a "Hilbert Space" diffraction tomography algorithm that is independent of measurement system geometry and the number of frequencies used in the reconstruction. The algorithm produces a full wave reconstruction without ray approximations. Focusing is achieved through mathematical back propagation.

\section{Results}

\section{Guided Waves}

A sample of alumina-epoxy-aluminum was constructed to study the propagation of guided waves in a metal-epoxy-ceramic structure. The sample has a transition from the multi-layer to a single layer structure. A notch was machined into the aluminum layer to simulate a crack in the bottom layer. The notch was approximately $30 \%$ through the wall of the aluminum. A schematic is shown in Figure 1. The transition is common to many practical applications such as lap joints in aircraft structures. Frequently, one is trying to inspect a single layer with access in the multi-layered section or vice versa. The transition will act as a reflector, since it is a disturbance of the waveguide.

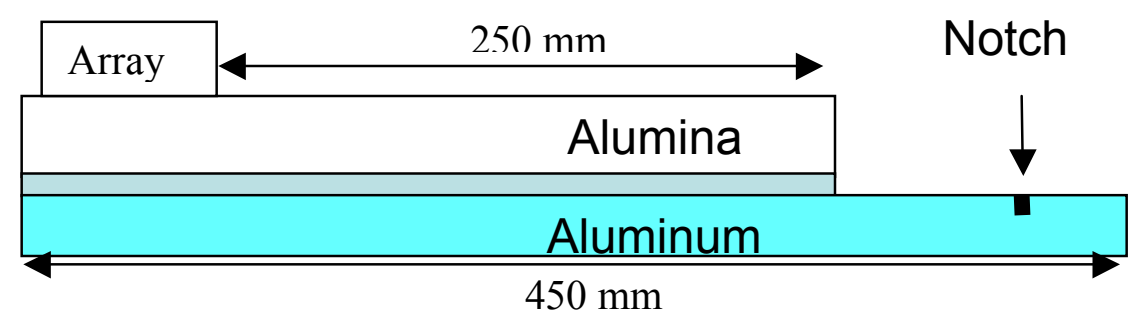

Figure 1. Schematic of an alumina-epoxy-Al sample used for experimental testing with a notch $1 \mathrm{~mm} \times 25 \mathrm{~mm}$ and $0.75 \mathrm{~mm}$ in depth.

Figure 2 shows the dispersion curves for an alumina-epoxy-aluminum structure with $6.35 \mathrm{~mm}$ of alumina, $0.1 \mathrm{~mm}$ epoxy, and $2 \mathrm{~mm}$ aluminum. The dispersion curves provide a basis for conducting experiments, interpreting data and designing sensors for optimal inspection capabilities. 


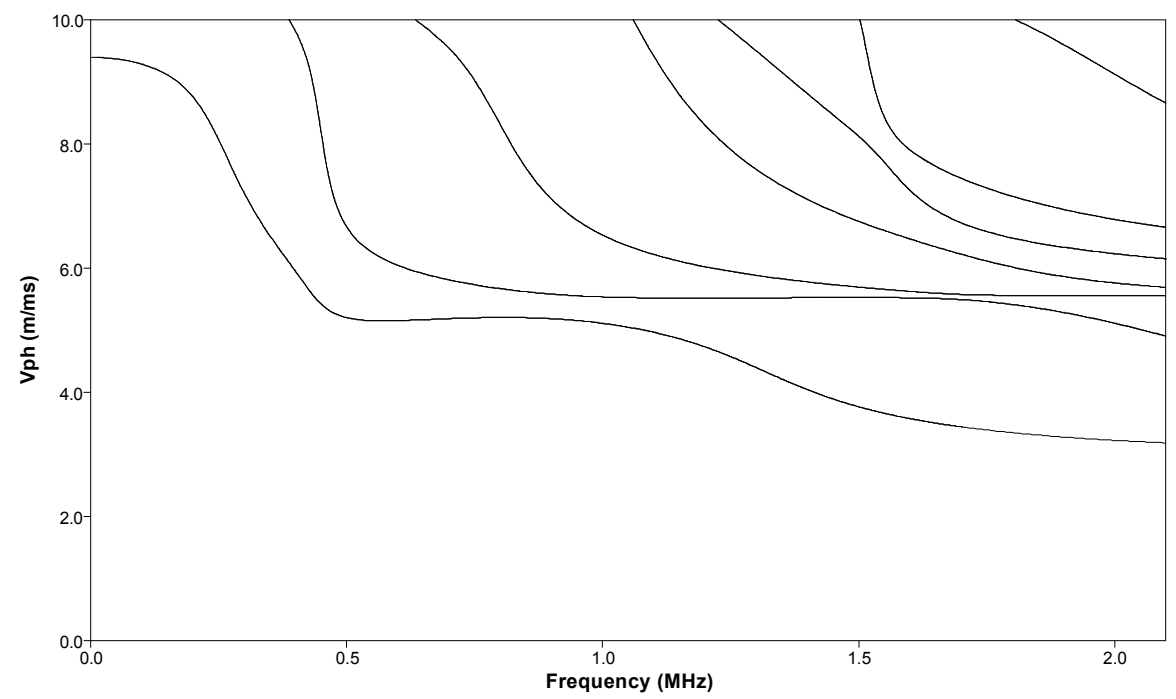

Figure 2. Phase velocity dispersion curves for an alumina-epoxy-aluminum structure with Alumina - $6.35 \mathrm{~mm}$, Epoxy - $0.1 \mathrm{~mm}$, Aluminum - $2 \mathrm{~mm}$.

A mode excited at $475 \mathrm{kHz}$ using a piezoelectric array and a phase velocity of $5.29 \mathrm{~km} / \mathrm{s}$ was found to produce good signal-to-noise. Array design analysis for mode excitation can be found in [3]. Figure 3 shows a sample RF waveform at $475 \mathrm{kHz}$ and the detection of the notch. A group velocity of $3.3 \mathrm{~km} / \mathrm{s}$ was observed and verified with the group velocity dispersion curves. Group velocity assists in identifying modes as well as localizing flaws.

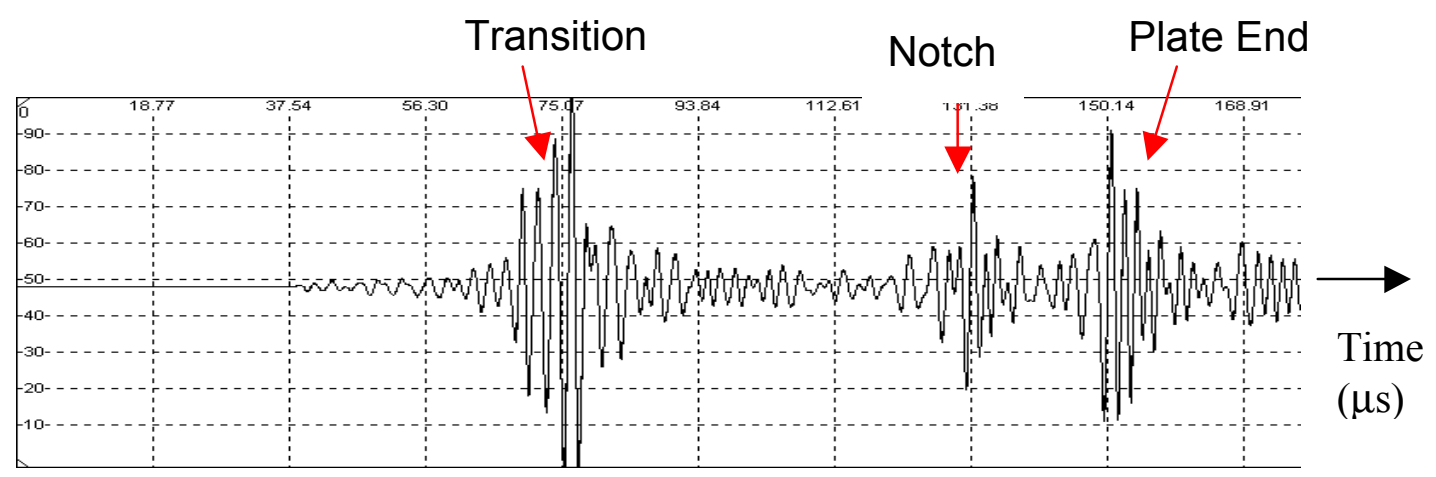

Figure 3. A sample RF waveform showing the detection of a notch in an alumina-epoxyaluminum multi-layer structure with a transition into a single layer.

Figure 4 shows the in-plane power for the mode at $475 \mathrm{kHz}$. The mode has sufficient energy at the surface to be excited and at the same time energy in the bottom layer. The result demonstrates that modes with energy in the bottom layer can be used to inspect the bottom layer in a multilayer structure [4]. 


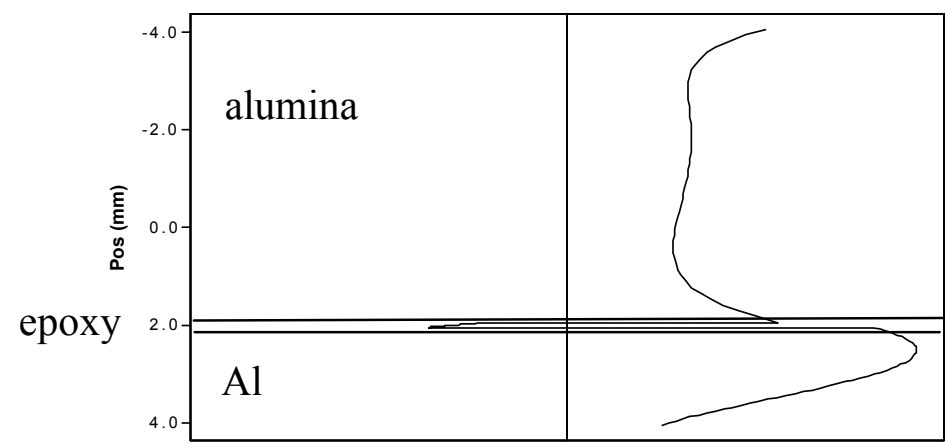

Normalized inplane power

Figure 4. Mode shape of in-plane power at $475 \mathrm{kHz}$ and $5.29 \mathrm{~km} / \mathrm{s}$ shows that the mode carries most of its energy in the bottom layer.

To further evaluate the quantitative detection abilities of the guided wave technique multilayer structures of Al-epoxy-steel were constructed with notches of depths $10 \%, 20 \%, 30 \%, 50 \%$, and $100 \%$ through-wall in the bottom Al layer. The structures were $900 \mathrm{~mm}$ in length and thicknesses were $3 \mathrm{~mm}$ for Al, $0.1 \mathrm{~mm}$ for epoxy, and $3 \mathrm{~mm}$ for steel. The guided wave technique showed sensitivity to detect the smallest $(10 \%)$ flaw as shown in Figure 5.
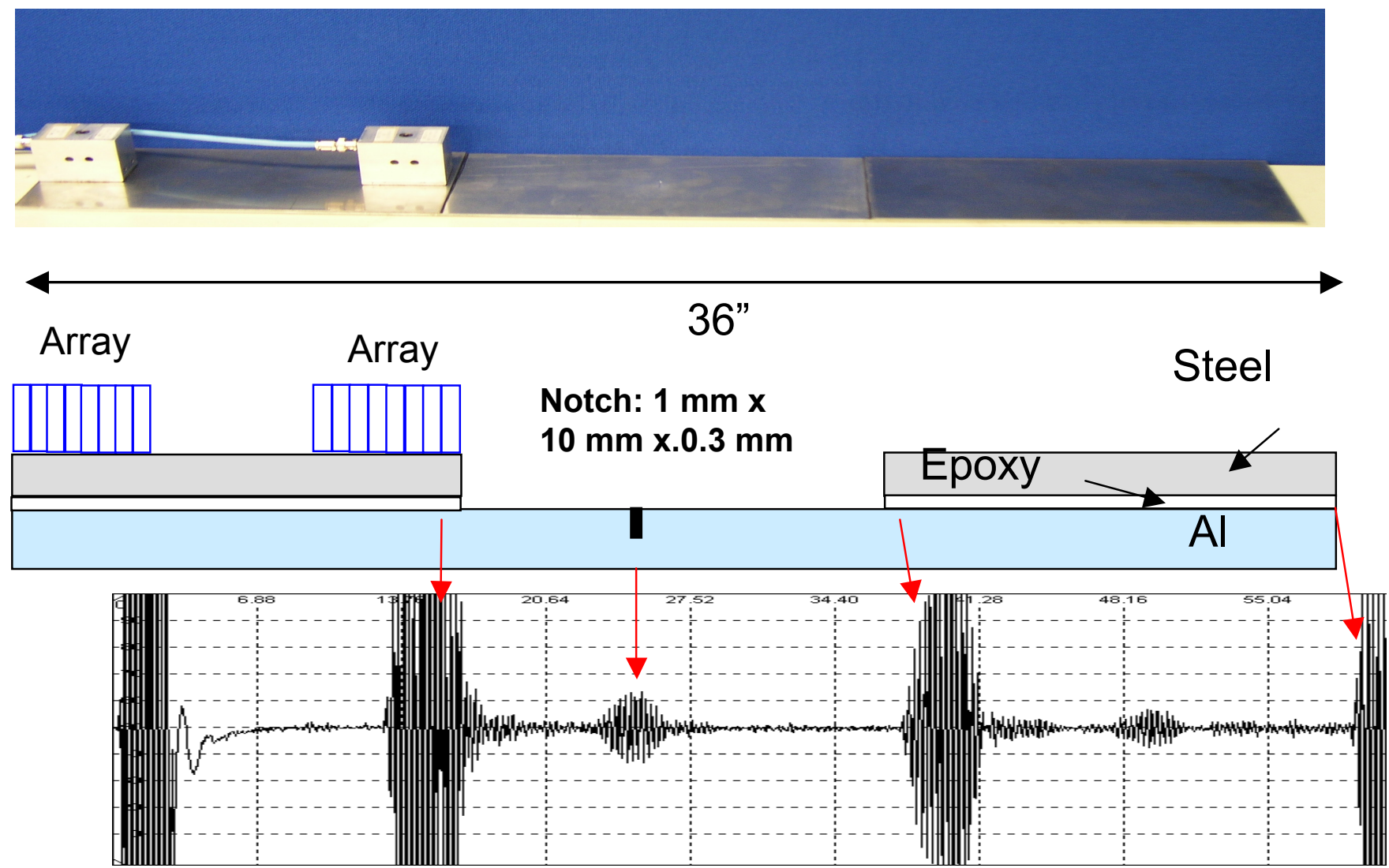

Figure 5. An EMAT array was used to detect a $10 \%$ through-wall $(0.3 \mathrm{~mm})$ notch in an aluminum-epoxy steel structure at $500 \mathrm{kHz}$. 
Next, curved multilayered structures were studied. A curved multilayer structure was manufactured using a stainless steel shell with $\mathrm{Al}$ layers bonded on to it with epoxy. An EDM notch, $0.75 \mathrm{~mm} \times 12 \mathrm{~mm} \times 0.75 \mathrm{~mm}$ (37\% through-wall), was placed at the pole of the shell. EMAT (electromagnetic acoustic transducer) arrays were used to excite and detect the guided waves. Figure 6 shows the waveform with the schematic of the shell. The EDM notch was detected with excellent signal-to-noise at $510 \mathrm{kHz}$. The experiment showed applicability of guided waves to curved multilayered structures.

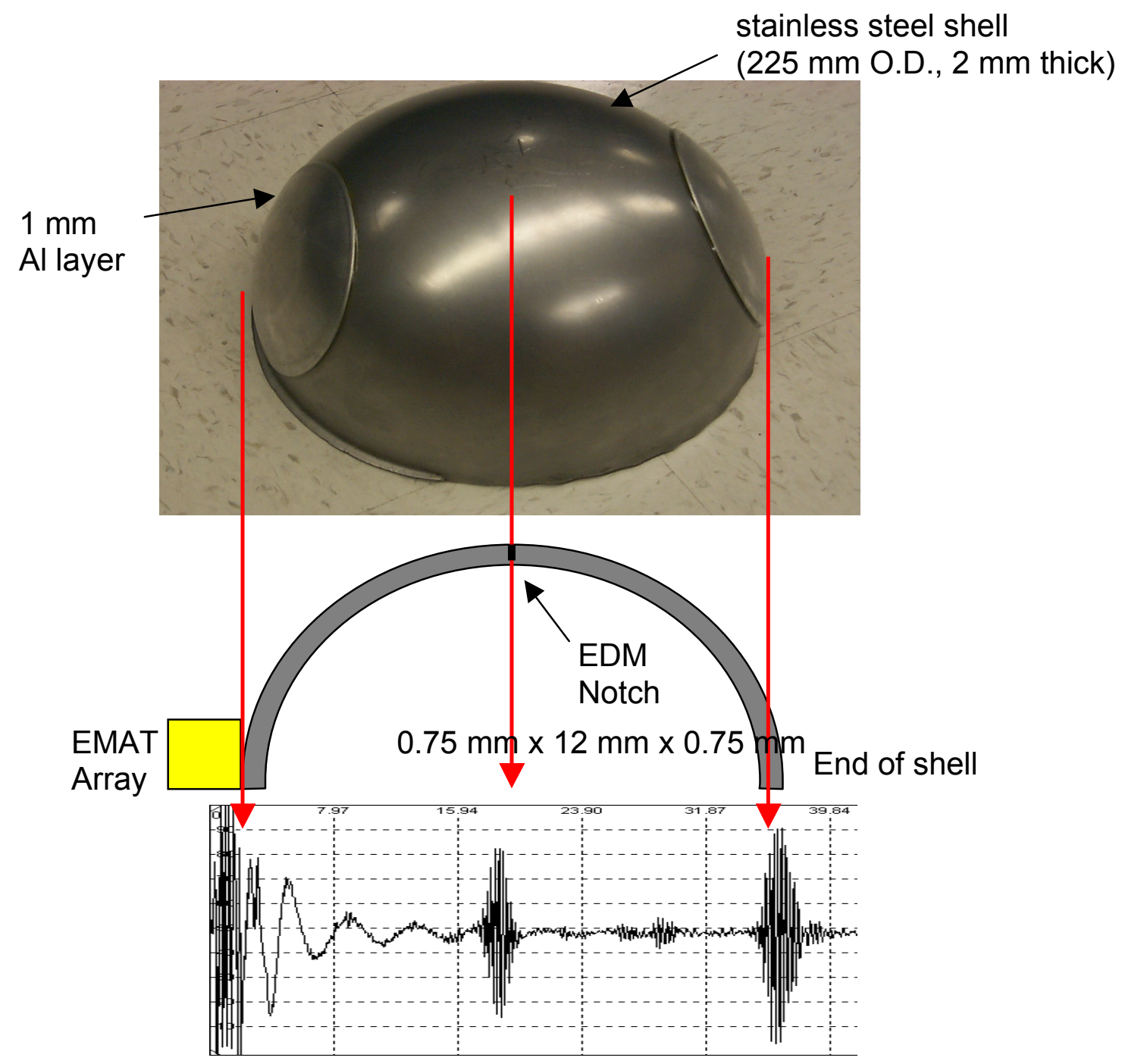

Time (proportional to distance)

Figure 6. A sample RF waveform shows the detection of an EDM notch using a $510 \mathrm{kHz}$ guided wave on a curved steel structure with Al layers bonded at the sides with epoxy.

Experimental studies were conducted on tubes to analyze the scalability and range of applicable curvatures. Additional experiments were conducted on a $0.5 \mathrm{~mm}$ thick and $3 \mathrm{~mm}$ O.D. stainless steel tube to show applicability to smaller curvatures. A through- 
wall crack and secondary cracking were created on a tube by bending it several times. A piezoelectric array excited a guided wave from one end of the tube and detected the crack (Figure 7). Eventually, we would like to scale down to sizes on the order of NIF targets.
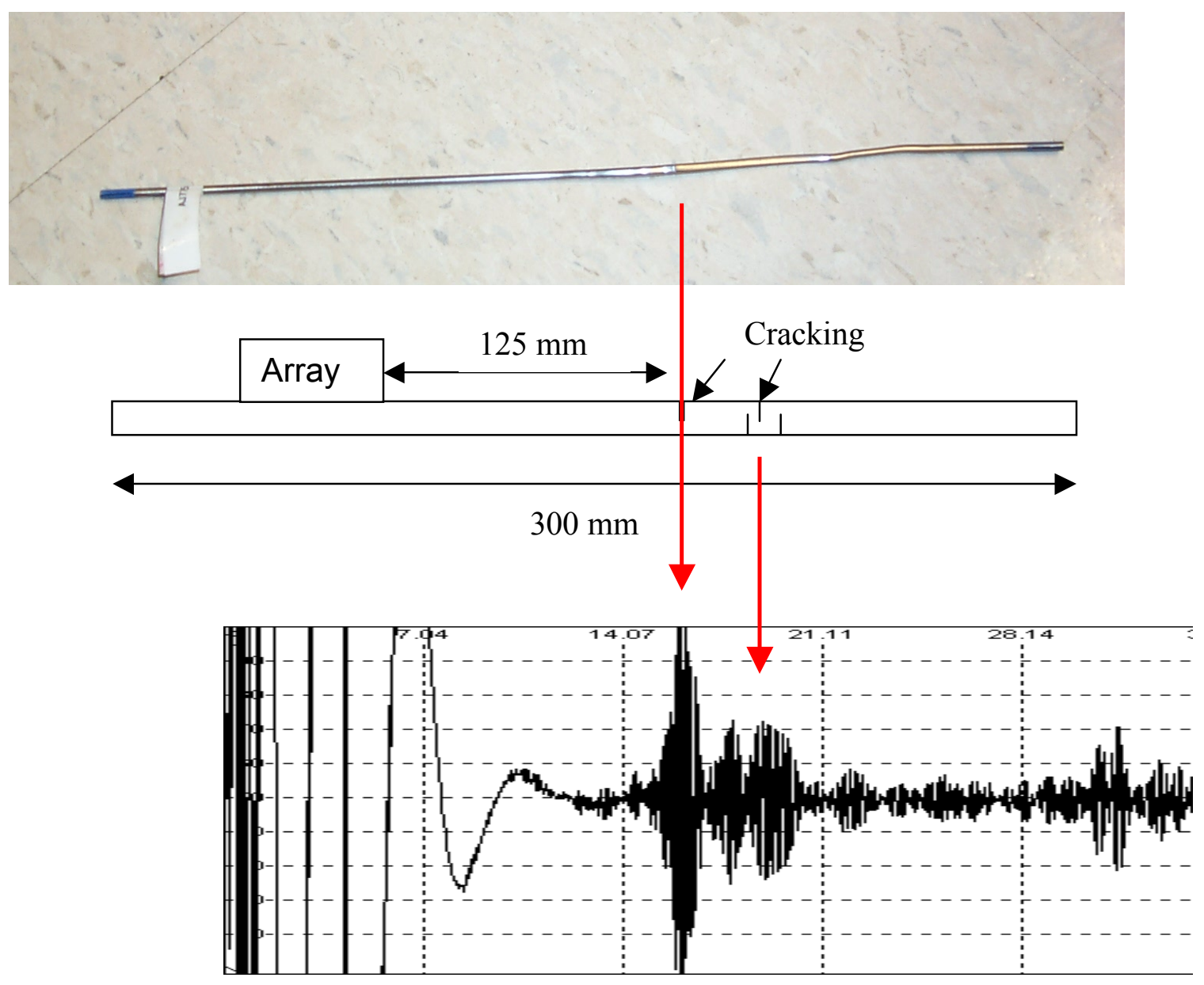

Time (proportional to distance)

Figure 7. Cracks in a $3 \mathrm{~mm}$ diameter tube were detected by a guided wave with the array at $125 \mathrm{~mm}$ from the largest through-wall crack at $980 \mathrm{kHz}$.

\section{Bulk Waves}

For bulk waves, an Al-Cu planar sample was constructed with a side-drilled hole. Experimental data was collected with a $5 \mathrm{MHz}$ ultrasonic array. The reconstruction process requires a multistatic data set representing the scattered field in the elastic region of interest. Multistatic data refers to the process in which one element of the array acts a transmitter to insonify the test region. The return time-series signals (echoes) are subsequently recorded on all of the elements; this process is repeated at all of the transmit-receive locations. To that end, we began by recording a background data set. The background data can be obtained from an identical part, or a region on the same part, that is free of defects. For our purposes, the background data were obtained by locating 
the transducer array over a region of the aluminum-copper plate far enough away from the hole to eliminate any reflections. A complete multistatic background data set was recorded. The array was then moved to a region over the flaw, and a second complete multistatic data set was recorded. This data set is referred to as the object data set. The complete multistatic scattered data set is then the difference between the object data set and the background data set. A bent-ray algorithm utilizing Fermat's principle corrects the time shifts in the ultrasonic data created by the multilayer structure for reconstruction. The image produced (Figure 8) shows an indication of a $2.5 \mathrm{~mm}$ side-drilled hole. The location of the hole was also accurately imaged. A detailed mathematical derivation of the algorithm can be found in [5]. Holes of diameters 0.75 and $1.5 \mathrm{~mm}$ were later tested. The bent-ray algorithm was able to detect down to a $0.75 \mathrm{~mm}$ flaw.

For the wave-based algorithm a planar multilayer Green function was derived and utilized in a Hilbert space diffraction tomography inversion algorithm developed by others that significantly simplify inversion [6,7]. The multilayer Green function accounts for the multilayer nature of the medium [8]. Data was reconstructed with the Hilbert space algorithm. The reconstruction of an Al-Cu sample with a $2.5 \mathrm{~mm}$ side drilled hole is shown in Figure 8. The wave-based algorithm was not able to image the smaller holes as the bent-ray was, but this algorithm is newer and requires more work.

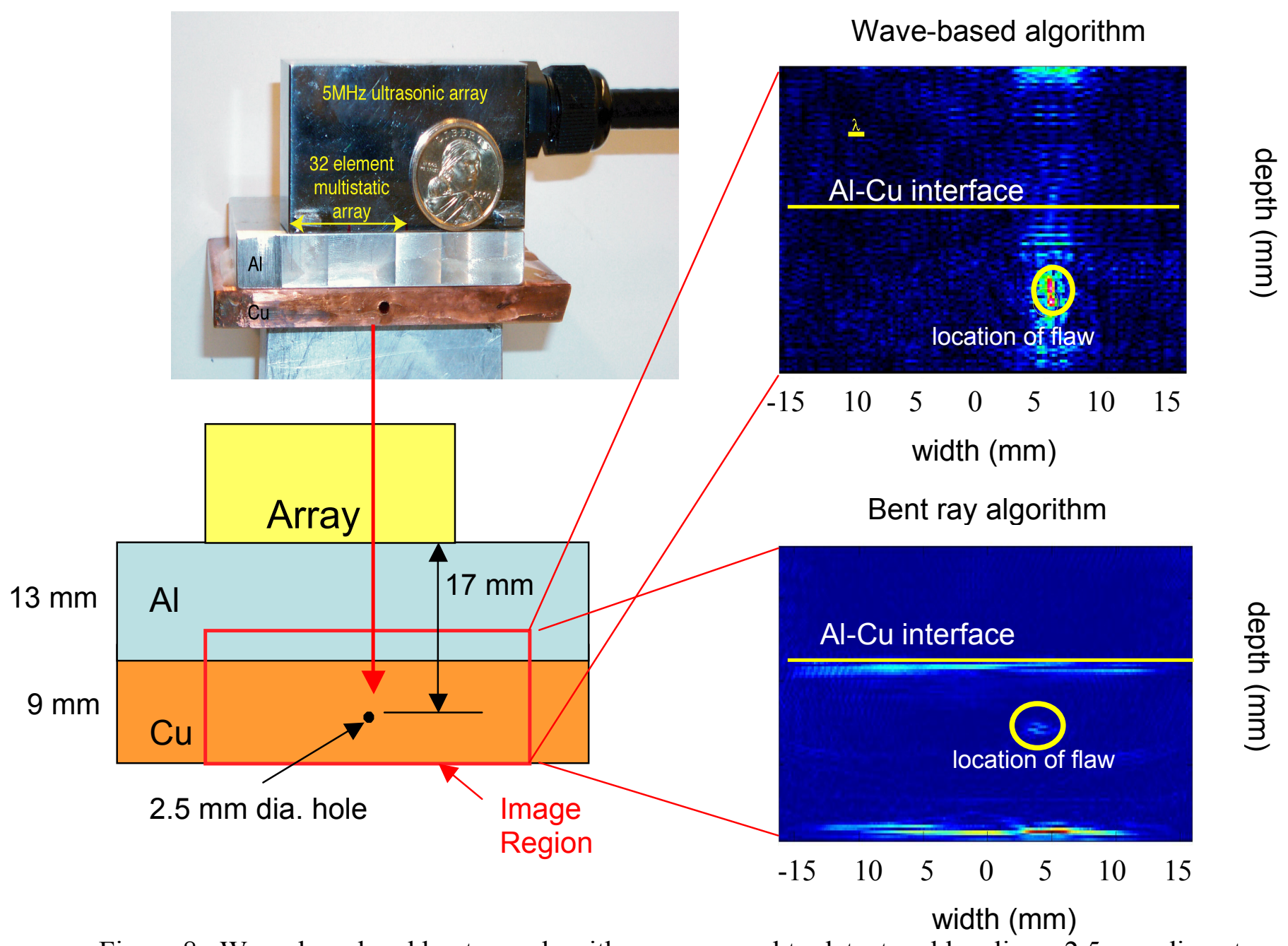

Figure 8. Wave-based and bent-ray algorithms were used to detect and localize a $2.5 \mathrm{~mm}$ diameter hole in an Al-Cu sample. 


\section{Summary}

A technique for inspecting multilayer structures was developed using ultrasonic arrays to selectively excite modes that preferentially inspect a desired layer. The technique was successfully demonstrated on flat and curved multilayered structures. Experiments on $3 \mathrm{~mm}$ diameter tubes showed the scalability of guided waves to smaller structures. Studies on notches of various depths showed sensitivity to be at $10 \%$ of the through-wall and possibly smaller.

Reconstruction algorithms were developed to overcome the difficulties of reflections and refractions at interfaces to enable accurate imaging of multilayered structures using bulk waves. Arrays were used to collect bulk wave data. A reconstruction based on Fermat's principle was shown to detect and accurately locate a flaw of $0.75 \mathrm{~mm}$ in diameter in an Al-Cu sample. A Hilbert space algorithm with an incorporated multilayer Green function was able to detect and locate a $2.5 \mathrm{~mm}$ diameter side-drilled hole in a reconstruction of an Al-Cu sample.

\section{Future work}

Guided wave work will look at incorporating time reversal techniques to improve resolution. Future bulk wave techniques will include shear waves and curvatures in the reconstruction algorithms to enhance signal-to-noise in images.

\section{Exit Plan}

We are in talks with several people at Los Alamos to utilize guided waves on weapons components. Guided waves also have potential application to problems in the life extension and advanced development and production technologies program. There is also potential for utilizing guided wave modes to inspect NIF targets. In addition, the planar multilayer diffraction tomography reconstruction algorithms may image density distributions in explosives for HEAF and Pantex. Also, the algorithms are applicable to shallow, near earth, environments to look for buried hazardous waste and covert transborder tunnels.

\section{References}

1. Quarry, M.J., Rose, J.L., "Multimodal Guided Wave Inspection of Piping Using Comb Transducers," Materials Evaluation, Vol. 57, No. 10, pp.1089-1093, October 1999.

2. Alleyne, D.N., Lowe, M.J.S., and Cawley, P., "The Excitation of Lamb Waves in Pipes Using Dry-Coupled Piezoelectric Transducers," Journal of Nondestructive Evaluation, Vol. 15, No. 1, pp 11-20, 1996. 
3. Quarry, M.J., "Phased Array Design Concepts for Lamb Wave Mode Tuning," Nondestructive Characterization of Materials, 2003, Vol. XI, pp. 81-87.

4. Quarry, M.J., "Guided Wave Inspection of Multilayered Structures," Review of Progress in Quantitative Nondestructive Evaluation, 2004, Vol. 23A, pp. 246-253.

5. Fisher, K.A., "Development of a quantitative multiview time domain imaging algorithm using a Fermat correction," to be published in the Journal of the Acoustical Society of America, 2005, Lawrence Livermore National Laboratory, Livermore, CA, UCRL-JRNL-209202.

6. Devaney, A.J., Dennison, M., "Inverse Scattering in Inhomogeneous Background Media," Inverse Problems, Vol. 19, pp 855-870, 2003.

7. Lehman, S.K., Norton, S.J., "Radial Reflection Diffraction Tomography," Journal of the Acoustical Society of America, Vol. 116(4), pp 2158-2172, 2004.

8. Lehman, S.K., "Hilbert Space Inverse Wave Imaging in a Planar Multilayer Environment," accepted for publication in the Journal of the Acoustical Society of America, 2005, Lawrence Livermore National Laboratory, Livermore, CA, UCRLJRNL-207624. 